\title{
Postcopulatory, prezygotic isolation: intraspecific and interspecific sperm precedence in Tribolium spp., flour beetles
}

\author{
THERESA ROBINSON, NORMAN A. JOHNSON† \& MICHAEL J. WADE†* \\ Southern Illinois University, Carbondale, IL 62901 and †Department of Ecology and Evolution, 1101 East 57th Street, \\ University of Chicago, Chicago, IL 60637, U.S.A.
}

\begin{abstract}
We investigated intraspecific and interspecific patterns of paternity in Tribolium castaneum, the flour beetle, by mating females either to pairs of conspecific males or to one conspecific and one heterospecific male of the closely related species, $T$. freemani. Females of both species store sperm in the spermatheca after copulation and postcopulatory, prezygotic reproductive isolation has been reported between this pair of species. When conspecific males of contrasting genotype were mated simultaneously to $T$. castaneum females, we observed extremely high levels of variation among females in the pattern of sperm precedence as shown by the offspring genotypes. In contrast, $T$. castaneum females mated simultaneously to a conspecific and a heterospecific male produced over 99 per cent conspecific progeny. When conspecific males were mated sequentially to $T$. castaneum females, within 3-7 days, all offspring were sired by the second male. In contrast, when a conspecific male was replaced with a $T$. freemani male, most females continued to produce only first-male, conspecific offspring for the next 10 days. When a heterospecific male was replaced with a conspecific male, T. castaneum females changed from producing hybrid to conspecific offspring within 3 days. We discuss the evolutionary implications of these findings.
\end{abstract}

Keywords: postmating reproductive isolation, prezygotic reproductive isolation, speciation, sperm precedence, Tribolium.

\section{Introduction}

Wade et al. (1994) observed postcopulatory, prezygotic reproductive isolation between two species of flour beetles, Tribolium castaneum and $T$. freemani. When females of either species are presented simultaneously with males of both species, they produce offspring sired only by the conspecific male although females of either species will produce near normal numbers of progeny when paired only with heterospecific males. Thus, the reproductive isolation appears to be facultative and we speculated that it involves some type of female choice of sperm or sperm competition within the female spermatheca (Wade et al., 1994). Females of both species are capable of storing sperm in the spermatheca after copulation and a single insemination will permit a female to lay fertile eggs throughout life (Sokoloff, 1974). In the recent literature, there are reports of similar postcopulatory but prezygotic isola-

*Correspondence. tion phenomena occurring in ground crickets (Howard \& Gregory, 1993) and two genera of grasshoppers (Hewitt et al., 1989; Bella et al., 1992). In these accounts, the authors suggested that the postcopulatory reproductive isolation could be an interaction between male sperm and the female reproductive tract that favoured conspecific over heterospecific sperm.

In intraspecific studies of sexual selection, female choice of mates via sperm recognition and male-male sperm competition have been demonstrated or postulated in several species of insects (Sivinski, 1980; Thornhill \& Alcock, 1983; Smith, 1984). In plants, pollen choice by differential pollen tube germination on the female stigmatic surface or differential pollen tube growth through the style has been hypothesized as a mechanism for ensuring offspring genetic quality, for ensuring outcrossing (Walsh \& Charlesworth, 1992), for promoting sexual selection in plants (Queller, 1983; Wilson \& Burley, 1983) or as a means of species recognition. Dobzhansky (1940), Lande (1981) and Heisler (1982) argued that the evolution of female mating 
preferences within species could be initiated as a response to selection for mate recognition to avoid heterospecific matings and attendent wastage of gametes.

In this paper, we report the results of further experimental studies of intraspecific and interspecific patterns of sperm precedence in $T$. castaneum females. In the intraspecific studies, we used visible genetic markers to establish paternity in the progeny of multiply inseminated females. Females were offered two conspecific males of contrasting genotype either sequentially or simultaneously in three treatments: (i) a week with a male of one genotype, which was then removed and replaced with a male of the alternate genotype; (ii) a reversal of the sequential order used in (i), and (iii) both genotypes of males offered simultaneously. We contrast the intraspecific patterns of paternity observed in the progeny of these conspecific matings with the patterns observed between species in heterospecific matings. We investigated the pattern of interspecific sperm precedence in $T$. castaneum females by substituting a heterospecific male from $T$. freemani for one of the genotypes of $T$. castaneum male in each of the three conspecific treatments.

\section{Materials and methods}

T. castaneum is a widespread, human-commensual flour beetle that is found in a variety of stored products (see Sokoloff (1974) for information about its general biology). $T$. freemani is a closely related species that is capable of producing abundant numbers of sterile hybrids in reciprocal crosses with $T$. castaneum (Hinton, 1948; Nakitta et al., 1981; Brownlee \& Sokoloff, 1988; Juan et al., 1993; Wade \& Johnson, 1994; Wade et al., 1994). These species are most easily identified by their large size difference: $T$. freemani is almost three times as massive as $T$. castaneum (Brownlee \& Sokoloff, 1988; Wade \& Johnson, 1994). The two species mate readily and mate choice experiments have revealed little, if any, premating reproductive isolation between these species (Wade et al., 1994).

We collected 54 virgin females from the c-SM ( $T$. castaneum-Standard Mixture) laboratory strain which were homozygous $b / b$, for an autosomal black bodycolour allele (see Wade, 1976, 1977, for information regarding c-SM). The black allele is segregating in the c-SM stock and is semidominant, so the three genotypes, $b / b,+/ \mathrm{b}$ and $+/+$, with the red wild-type coloration, can be distinguished by eye (Wade \& Goodnight, 1991). These females were divided randomly into six groups of nine females and each group was subjected to one of six different experimental treat- ments: three conspecific treatments and three heterospecific treatments.

In the first conspecific treatment, each virgin c-SM $b / b$ female was paired with a single c-SM male, homozygous for the wild-type body colour allele $(+/+)$, in $8 \mathrm{~g}$ of standard medium husbanded in a darkened incubator maintained at $29^{\circ} \mathrm{C}$ and approximately 70 per cent relative humidity (Wade \& Johnson, 1994). After 7 days, the male was removed, the female was transferred to a new vial of medium and paired with a second c-SM male, homozygous for the $b / b$ genotype (Table 1). At each of days 9 (after 2 days with the second male), 12,14 and 18, the pair of beetles was removed and transferred to a fresh vial of medium. The progeny produced in each of the six vials per female were censused at maturity and scored by eye for bodycolour genotype. The homozygous $b / b$ progeny were sired by the second male and the $+/ b$ heterozygous progeny were sired by the first male. A similar procedure was followed with the second treatment group of $9 \mathrm{c}-\mathrm{SM} b / b$ females, except that the order of the male genotypes was reversed. We refer to these as the two conspecific sequential treatments.

In the two heterospecific sequential treatments, $T$. freemani $+1+$ males were used instead of the c-SM $+1+$ males. That is, a c-SM $b / b$ female was paired with either a c-SM $b / b$ male or a $T$. freemani $+1+$ male (we indicate the genotype of $T$. freemani males as homozygous wild-type, $+1+$, because hybrids carrying the $b$ allele from $T$. castaneum are similar in coloration to the $+/ b T$. castaneum heterozygotes (Brownlee \& Sokoloff, 1988)). As in the two conspecific sequential treatments above, after 7 days the first male was removed and replaced with a male of the opposite genotype (species). Subsequently, each pair of adults was transferred four times to new medium following the same schedule as above. At maturity, the hybrid offspring sired by the $T$. freemani male are clearly distinguishable by eye both by body coloration and by body size.

We also set up a heterospecific simultaneous treatment in which each of $9 \mathrm{c}-\mathrm{SM} b / b$ females was paired simultaneously with both a c-SM $b / b$ male and a $T$. freemani $+1+$ male (similar to the treatment CF-C in Wade et al. (1994)). A similar conspecific simultaneous treatment was set up in which each of nine females was paired simultaneously with both a c-SM $b / b$ male and a $\mathrm{c}-\mathrm{SM}+1+$ male (see Table 1$)$. In both simultaneous treatments, each group of three adults was transferred to a new vial of medium on the schedule described above for the sequential treatments. There was a total of 270 vials set up (six treatments $\times$ nine females per treatment $\times$ five vials per female $=270$ vials . 
Table 1 Experimental design

\begin{tabular}{|c|c|c|c|c|}
\hline Treatment & Female & \multicolumn{2}{|c|}{ Two males } & Trios \\
\hline \multicolumn{5}{|c|}{ Simultaneous treatments } \\
\hline $\begin{array}{l}\text { 1. Conspecific } \\
\text { simultaneous }\end{array}$ & $c b / b$ & \multicolumn{2}{|c|}{$\mathrm{c} b / b$ and $\mathrm{c}+/+$} & 9 \\
\hline $\begin{array}{l}\text { 2. Heterospecific } \\
\text { simultaneous }\end{array}$ & $\mathrm{c} b / b$ & \multicolumn{2}{|c|}{$\mathrm{c} b / b$ and $\mathrm{F}+1+$} & 9 \\
\hline Treatment & Female & 1st male & 2nd male & Pairs \\
\hline \multicolumn{5}{|c|}{ Sequential treatments } \\
\hline $\begin{array}{l}\text { 1. Conspecific } \\
\text { sequential }\end{array}$ & $c b / b$ & $c b / b$ & $\mathrm{c}+/+$ & 9 \\
\hline $\begin{array}{l}\text { 2. Conspecific } \\
\text { sequential }\end{array}$ & $c b / b$ & $c+1+$ & c $b / b$ & 9 \\
\hline $\begin{array}{l}\text { 3. Heterospecific } \\
\text { sequential }\end{array}$ & $\mathrm{c} b / b$ & $\mathrm{c} b / b$ & $\mathrm{~F}+1+$ & 9 \\
\hline $\begin{array}{l}\text { 4. Heterospecific } \\
\text { sequential }\end{array}$ & $\mathrm{c} b / b$ & $\mathrm{~F}+1+$ & $c b / b$ & 9 \\
\hline
\end{tabular}

\section{Results}

The results observed in the heterospecific simultaneous treatment were similar to those reported in Wade et al. (1994). When a T. castaneum female was paired simultaneously with a conspecific male and a $T$. freemani male, the conspecific male sired virtually all (more than 99 per cent) of the progeny (Fig. 1). In contrast, when a $T$. castaneum female is paired simultaneously with two conspecific males of different genotype, each male sired some of the progeny (Fig. 1). It is clear from Fig. 1 that the c-SM $+1+$ males sired a greater fraction, 65.3 per cent (885 of 1356 total offspring), of the offspring produced than did the c-SM $b / b$ males (34.7 per cent). There was, however, considerable heterogeneity among females and among transfers in the proportion of offspring sired by the $\mathrm{c}-\mathrm{SM}+1+$ males. The percentage progeny sired by the $\mathrm{c}-\mathrm{SM}+1+$ male ranged from 0 to 97.3 per cent across females ( $G$-test of heterogeneity among females, $G_{\mathrm{H}}=480.32,8$ d.f., $P \lll 0.0001$ over all transfers $)$ and ranged between 74.4 per cent and 50.7 per cent among transfers ( $G$-test of heterogeneity among transfers, $G_{\mathrm{H}}=66.76,3$ d.f., $\left.P \ll 0.0001\right)$. This heterogeneity among females in conspecific paternity is in marked contrast to the uniformity among females observed in the heterospecific paternity pattern where the c-SM $b /$ $b$ males sired in excess of 99.8 per cent of all offspring (Fig. 1). (Frequent bouts of interspecific copulation were observed in these treatments at the time of transfer to new medium).

There was no significant heterogeneity between sequential conspecific treatments owing to the order of

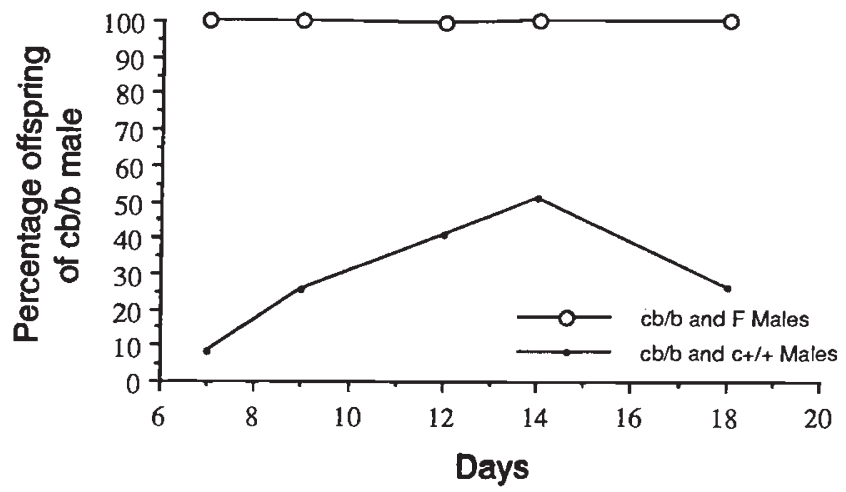

Fig. 1 Percentage of offspring produced by c-SM $b / b$ females that were sired by the c-SM $b / b$ male when in competition with either a $T$. freemani male ( $\mathrm{F}$, open circles) or a c-SM $+/+$ male (closed circles).

presentation of the male genotypes. In the conspecific sequential treatments, regardless of the first male's genotype, the pattern of paternity changed from 100 per cent offspring of the first male to more than 99 per cent paternity of the second male (Fig. $2 a$ and b). At all transfers where the fraction of progeny sired by one genotype was between 10 and 90 per cent, we tested for heterogeneity among the females in the rate at which offspring paternity changed from the initial to the final pattern (transfer 2 in Fig. 2a and transfers 2 and 3 in Fig. $2 b$ ). There was significant heterogeneity among females within treatments in every case (three $G$-tests, $\left.47<G_{\mathrm{H}}<116, P \ll 0.0001\right)$ but not between treatments.

In the heterospecific sequential treatments, when a $T$. freemani male was replaced with a c-SM $b / b$ male, paternity of offspring changed within one transfer to 97.1 per cent conspecific paternity, followed by 100 per cent conspecific paternity at all subsequent transfers. There was no heterogeneity among females in this pattern (Fig. 2b). When a c-SM $b / b$ male was replaced with a $T$. freemani male, paternity of offspring by the absent conspecific male remained in excess of 91 per cent for the next 6 days before falling to 75.2 per cent by 9 days after replacement. However, there was considerable heterogeneity among c-SM $b / b$ females at the final transfer ( $G$-test of heterogeneity among females, $G_{\mathrm{H}}=453.66,6$ d.f., $\left.P \lll 0.0001\right)$. The majority of females (four of seven surviving to day 16 and producing offspring) continued to produce only conspecific progeny, two produced intermediate frequencies of hybrid offspring $(59.5$ per cent and 83.15 per cent) and one produced only hybrid progeny (100 per cent) at that time. (The four females producing conspecific progeny averaged 87.8 $(\mathrm{SE}=28.98)$ offspring per female while the three females producing hybrid offspring averaged 50.7 $(\mathrm{SE}=6.01)$. $)$ 

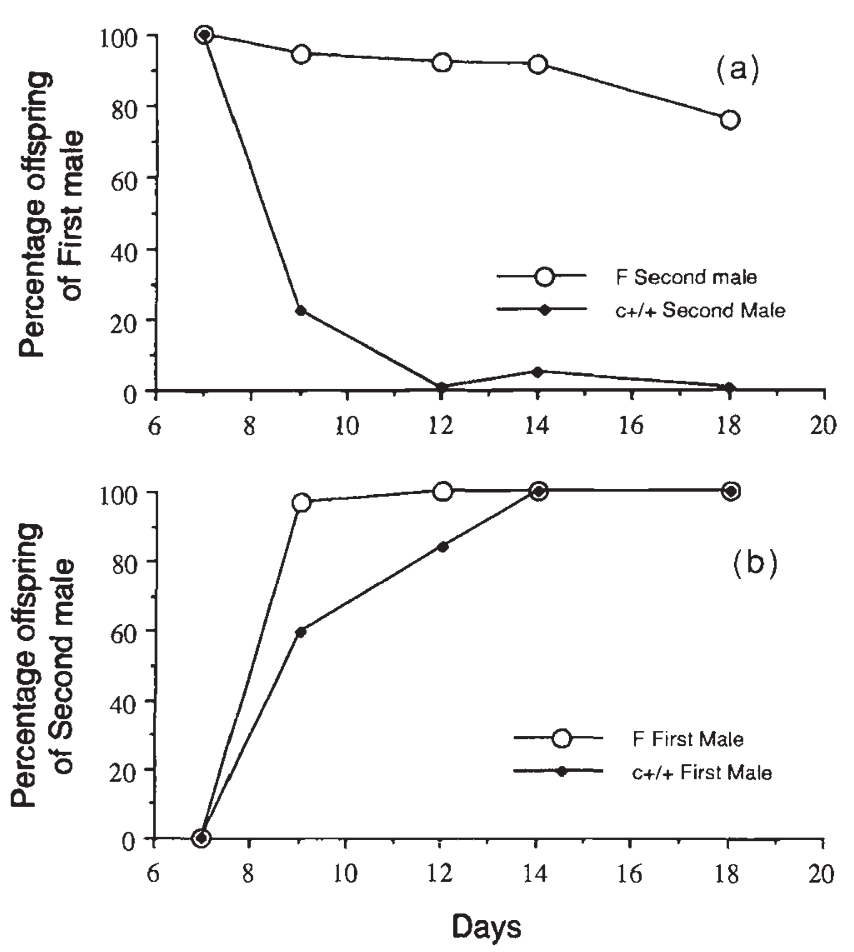

Fig. 2 Percentage of offspring sired by a c-SM $b / b$ male when it is the first of two males paired with a c-SM $b / b$ female ( $\mathrm{a}$ ) or the second of two males paired with a c-SM $b / b$ female (b, bottom graph). Graph (a) shows that the sperm of the c-SM $b / b$ male are more rapidly replaced by that of a conspecific male $(c+1+)$ than that of a heterospecific male (F). Graph (b) shows that a c-SM $b / b$ male more rapidly displaces the sperm of a heterospecific male $(F)$ than a conspecific male $(\mathrm{c}+1+)$, although both are eventually displaced.

\section{Discussion}

We show the following patterns of interspecific sperm precedence observed in fertilization in $T$. castaneum females:

1 in simultaneous competition, conspecific sperm are used instead of heterospecific sperm to fertilize eggs (Fig. 1; Wade et al., 1994),

2 conspecific sperm have precedence over heterospecific sperm even in the absence of the conspecific male (Fig. 2a),

3 the sperm precedence of a second T. castaneum male is established more rapidly when he replaces a heterospecific male than when he replaces a conspecific male (Fig. 2b), and

4 there is much greater variability in sperm precedence when the two competing males are conspecifics than when a conspecific male is competing with a heterospecific male either simultaneously (Fig. 1) or sequentially (Fig. 2a and b). All four results reported above using the black locus as a visible marker are similar to those observed in a smaller, pilot study using the sex-linked recessive eye colour mutation, pearl ( $\mathrm{T}$. Robinson, unpublished data).

The extensive literature on patterns of sperm precedence in Drosophila melanogaster is reviewed by Gromko et al. (1984) and for insects in general by Gwynne (1984). However, these reviews fail to discuss the possible role that these intraspecific patterns of sperm precedence might play in the early stages of the evolution of postcopulatory reproductive isolation and hence speciation. The large among-pair variance in paternity patterns within species may contribute to the rapid evolution of divergence among species in traits related to sperm predominance. Recent reports in some insects (Hewitt et al., 1989; Bella et al., 1992; Howard \& Gregory, 1993; Wade et al., 1994) indicate that postcopulatory, prezygotic paternity selection can serve as a mechanism of species recognition. In flour beetles, sperm are immotile at the time of ejaculation and appear to become active in the spermatheca of the female (M. Wade, personal observation). It is possible that the interaction of male sperm and a female activation factor(s) serves as a mechanism of postcopulatory, prezygotic species recognition.

One mechanism of postcopulatory, prezygotic reproductive isolation is the removal of sperm from previous inseminations from the female's reproductive tract by later mating males while in copula. The phenomenology we have documented could arise if conspecific males were better able to remove the sperm from the female spermatheca than were heterospecific males and/or if heterospecific sperm were more easily removed than conspecific sperm. Gage (1992) has shown that males of the related beetle, Tenebrio molitor, were able to remove the sperm of rival males. We think that this is unlikely to occur in Tribolium, however, because mating duration is very short, generally less than 1 minute (Park, 1933; Sokoloff, 1974) and Tribolium males lack the sperm-removing penile spines found in Tenebrio males.

\section{Acknowledgements}

We thank Michael McNaughton and Ora Lucas for technical assistance and Mathew Leibold, Alan Molumby and Mohamed Noor for discussion of the work. This work was supported by National Institutes of Health grant (GM 22523) to M.J.W.

\section{References}

BELLA, J. L., BUTLIN, R. K., FERRIS, C. AND HEWITT, G. M. 1992. Asymmetrical homogamy and unequal sex ratios from reciprocal mating-order crosses between Chorthippus parallelus subspecies. Heredity, 68, 345-352. 
BROWNLEE, A. AND SOKOLOFF, A. 1988. Transmission of Tribolium castaneum (Herbst) mutants to $T$. castaneum $-T$. freemani (Hinton) hybrids (Coleoptera: Tenebrionidae). $J$. Stored Prod. Res., 24, 145-150.

DOBZHANSKY, TH. 1940. Speciation as a stage in evolutionary divergence. Am. Nat., 74, 312-321.

GAGE, M. J. G. 1992. Removal of rival sperm during copulation in a beetle, Tenebrio molitor. Anim. Behav., 44, 587-589.

GROMKo, M. H., GILBERT, D. G. AND RICHMOND, R. C. 1984. Sperm transfer and use in the multiple mating system of Drosophila. In: Smith, R. L. (ed.) Sperm Competition and the Evolution of Animal Mating Systems, pp. 371-426. Academic Press, Orlando, FL.

GWYNNE, D. T. 1984. Male mating effort, confidence of paternity, and insect sperm competition. In: Smith, R. L. (ed.) Sperm Competition and the Evolution of Animal Mating Systems, pp. 117-149. Academic Press, Orlando, FL.

HEISLER, 1. L. 1982. Genetical Studies of Mating Preference. Ph. D. Thesis, University of Chicago, p. 139.

HEWITT, G. M., MASON, P. AND NICHOLS, R. A. 1989. Sperm precedence and homogamy across a hybrid zone in the alpine grasshopper, Podisma pedestris. Heredity, 62, 343-353.

HINTON, H. E. 1948. A synopsis of the genus Tribolium MacLeay, with some remarks on the evolution of its species-groups (Coleoptera: Tenebrionidae). Bull. Ent. Res., 39, 13-55.

HOWARD, D. J. AND GREGORY, P. G. 1993. Post-insemination signalling systems and reinforcement. Phil. Trans. R. Soc. Lond, B., 340, 231-236.

JUAN, C., VAZQUEZ, P., RUBIO, J. M., PETTITPIERRE, E. AND HEWITT, G. M. 1993. Presence of highly repetitive DNA sequences in Tribolium flour beetles. Heredity, 70, 1-8.

LANDE, R. 1981. Models of speciation by sexual selection on polygenic traits. Proc. Natl. Acad. Sci. U.S.A., 78, 3721-3725.

NAKAKITA, H., IMURA, O. AND WINKS, R. G. 1981. Hybridization between Tribolium freemani (Hinton) and Tribolium castaneum (Herbst) and some preliminary studies on the biology of Tribolium freemani (Coleoptera: Tenebrionidae). Appl. Ent. Zool., 16, 209-215.
PARK, T. 1933. Studies in population physiology. II. Factors regulating initial growth of Tribolium confusum populations. Exp. Zool., 65, 17-42.

QUELLER, D. 1983. Sexual selection in a hermaphroditic plant. Nature, 305, 706-707.

SIVINSKı, J. 1980. Sexual selection and insect sperm. Fla. Entomologist, 63, 99-111.

SMITH, R. L. (ED.) 1984. Sperm Competition and the Evolution of Animal Mating Systems. Academic Press, Orlando, FL.

soKolofF, A. 1974. The Biology of Tribolium, vol. 2. Clarendon Press, Oxford.

THORNHILL, R. AND ALCOCK, J. 1983. The Evolution of Insect Mating Systems. Harvard University Press, Cambridge, MA.

WADE, M. J. 1976. Group selection among laboratory populations of Tribolium. Proc. Natl. Acad. Sci. U.S.A., 73, 4604-4607.

WADE, M. J. 1977. An experimental study of group selection. Evolution, 31, 134-153.

WADE, M. J. AND GOODNIGHT, C. J. 1991. Wright's shifting balance theory: an experimental study. Science, 253, 1015-1018.

WADE, M. J. AND JOHNSON, N. A. 1994. Reproductive isolation between two species of flour beetles, Tribolium castaneum and $T$. freemani: variation within and among geographical populations of $T$. castaneum. Heredity, 72, 155-162.

WADE, M. J., PATTERSON, H., CHANG, N. W. AND JOHNSON, N. A. 1994. Postcopulatory, prezygotic isolation in flour beetles. Heredity, 72, 163-167.

WALSH, N. E. AND CHARLESWORTH, D. 1992. Evolutionary interpretations of differences in pollen tube growth rates. $Q$. Rev. Biol., 67, 19-36.

wilson, M. F. AND Burley, N. 1983. Mate Choice in Plants. Princeton University Press, Princeton, NJ. 